\title{
Antimicrobial effects of liquid anesthetic isoflurane on Candida albicans
}

\author{
Viachaslau M Barodka1, Edward Acheampong ${ }^{\dagger 2}$, Garry Powell ${ }^{\dagger 1}$, \\ Ludmila Lobach $^{\dagger 2}$, David A Logan ${ }^{3}$, Zahida Parveen ${ }^{2}$, Valerie Armstead ${ }^{1}$ and \\ Muhammad Mukhtar*4
}

\begin{abstract}
Address: ${ }^{1}$ Anesthesiology Program For Translational Research, Department of Anesthesiology, Thomas Jefferson University, Philadelphia, Pennsylvania, USA, ${ }^{2}$ Division of Infectious Diseases, Department of Medicine, Thomas Jefferson University, Philadelphia, Pennsylvania, USA, ${ }^{3}$ Department of Biological Sciences, Clark Atlanta University, Atlanta, Georgia, USA and ${ }^{4}$ Department of Microbiology and Immunology, Institute for Molecular Medicine and Infectious Disease, Drexel University College of Medicine, Philadelphia, Pennsylvania, USA

Email: Viachaslau M Barodka - vbarodka@yahoo.com; Edward Acheampong - eacheamp@yahoo.com;

Garry Powell - garry.powell@jefferson.edu; Ludmila Lobach - lobachluda@yahoo.com; David A Logan - loganda77@hotmail.com;

Zahida Parveen - zahida.parveen@jefferson.edu; Valerie Armstead - valerie.armstead@jefferson.edu;

Muhammad Mukhtar* - muhammad.mukhtar@drexelmed.edu

* Corresponding author †Equal contributors
\end{abstract}

Published: 09 November 2006

Journal of Translational Medicine 2006, 4:46 doi:10.1 186/1479-5876-4-46
Received: II September 2006

Accepted: 09 November 2006

This article is available from: http://www.translational-medicine.com/content/4/I/46

(c) 2006 Barodka et al; licensee BioMed Central Ltd.

This is an Open Access article distributed under the terms of the Creative Commons Attribution License (http://creativecommons.org/licenses/by/2.0), which permits unrestricted use, distribution, and reproduction in any medium, provided the original work is properly cited.

\begin{abstract}
Candida albicans is a dimorphic fungus that can grow in yeast morphology or hyphal form depending on the surrounding environment. This ubiquitous fungus is present in skin and mucus membranes as a potential pathogen that under opportunistic conditions causes a series of systemic and superficial infections known as candidiasis, moniliasis or simply candidiasis. There has been a steady increase in the prevalence of candidiasis that is expressed in more virulent forms of infection. Although candidiasis is commonly manifested as mucocutaneous disease, life-threatening systemic invasion by this fungus can occur in every part of the body. The severity of candidal infections is associated with its morphological shift such that the hyphal morphology of the fungus is most invasive. Of importance, aberrant multiplication of Candida yeast is also associated with the pathogenesis of certain mucosal diseases. In this study, we assessed the anti-candidal activity of the volatile anesthetic isoflurane in liquid form in comparison with the anti-fungal agent amphotericin $B$ in an in vitro culture system. Exposure of $C$. albicans to isoflurane ( $0.3 \%$ volume/volume and above) inhibited multiplication of yeast as well as formation of hyphae. These data suggest development of potential topical application of isoflurane for controlling a series of cutaneous and genital infections associated with this fungus. Elucidiation of the mechanism by which isoflurane effects fungal growth could offer therapeutic potential for certain systemic fungal infections.
\end{abstract}

\section{Background}

Candida albicans is an opportunistic fungal pathogen mainly causing infections among immunocompromised individuals [1]. Infections associated with this fungus range from superficial mycoses to life threatening sys- temic candidiasis, which involve various body organs and invasive mucosal disorders. A wide spectrum of infections associated with C. albicans are ascribed to the capability of this fungus to switch between unicellular budding yeast to multicellular, filamentous mycelial or hyphal form [2]. 
Several studies in the past have reported that the hyphal form of C. albicans is more invasive compared with budding yeast [2-4]. This hypothesis has been further strengthened in a rodent model of fungal infection where C. albicans strains unable to form hyphae were essentially avirulent [5]. As such, there has always been a need for an antifungal agent that can both prevent the formation of hyphae as well as destroy both yeast and hyphal morphologies of this fungus.

Isoflurane is a halogenated volatile anesthetic and its gaseous form has shown antibacterial activity against one of the most common bacterial pathogens in hospitalacquired pneumonia Pseudomonas aeruginosa [6]. One study on the other hand did not find any antibacterial activity of isoflurane against Staphylococcus aureus and Escherichia coli [7]. Prospects for clinical usage of the gaseous isoflurane as an antibacterial agent have been hampered due to adverse systemic effects and occupational hazards $[8,9]$. Encouraged by our initial data showing that the gaseous form of isoflurane inhibits C. albicans morphogenesis (formation of hyphae from yeast cells, unpublished data), we analyzed the anti-candidal potential of volatile liquid isoflurane. C. albicans in vitro cultures were treated with various concentrations of isoflurane in semianaerobic as well as aerobic environments. Growth behavior and morphological changes were then assessed. For our initial studies, semi-anaerobic study design was essentially based on the volatile nature of the isoflurane with the challenge to maintain uniform concentrations of volatile anesthetic in the in vitro culture. Moreover, like several other fungi, C. albicans is a facultative anaerobe and can fulfill energy requirements either in the presence of oxygen or in the absence via fermentation [10]. Intriguingly, aerobic cultures of the yeast treated with isoflurane also revealed inhibitory effects on both yeast multiplication and formation of hyphae. We observed that isoflurane could completely abolish formation of hyphae in $C$. albicans, a step essential for the pathogenesis of this fungus in vivo. A concentration of $0.5 \%$ isoflurane $(\mathrm{v} / \mathrm{v})$ showed fungicidal activity equivalent to amphotericin $\mathrm{B}$, $4.0 \mu \mathrm{g} / \mathrm{ml}$ a concentration that has been previously reported as fungicidal [11]. Moreover, C. albicans treated with lower concentrations of isoflurane $(0.1-0.4 \%)$ showed diminished growth. These findings are highly significant, as this approach will provide a platform for a systematic study of fungal morphogenesis at the molecular level. Moreover, isoflurane in gaseous form is a widely used anesthetic approved by the U.S. Food and Drug Administration (FDA). Although usage of the gaseous form of isoflurane to treat systemic infections may not be feasible due to its anesthetic actions as well as adverse cardiovascular effects at high concentrations, however, possibilities exist for manipulation of the antimicrobial potentials of isoflurane. This is the first report revealing liquid isoflurane-mediated control of $C$. albicans growth and morphogenesis in a concentration-dependent manner. These findings suggest that there may be an opportunity to develop topical microbicides based on creative formulations that modulate the release of isoflurane. This strategy may provide a means for controlling Candida and related superficial infections that affect millions of immunocompromised and healthy individuals worldwide.

\section{Results \\ Effects of liquid isoflurane on the metabolic activity of C. albicans}

In our pilot studies we evaluated the effects of clinically relevant concentrations of gaseous isoflurane on C. albicans morphogenesis and observed an inhibitory effect on the formation of true hyphae from the yeast form of this fungus (data not illustrated). Encouraged by our initial observations we further analyzed the effects of various concentrations of liquid isoflurane on the morphogenesis and growth of C. albicans.

Isoflurane concentrations of $0.1 \%(\mathrm{v} / \mathrm{v})$ and higher significantly inhibited metabolic activity of C. albicans ( $\mathrm{p}<$ 0.001 compared to control), however, a concentration of $0.4-0.5 \%(\mathrm{v} / \mathrm{v})$ was critical in this study to achieve inhibitory activity similar to that of amphotericin B $(4.0 \mu \mathrm{g} . \mathrm{mL})$ (Fig 1B). Concentrations of liquid isoflurane less than $0.1 \%(\mathrm{v} / \mathrm{v})$ did not significantly inhibit metabolic activity (determined by the fungi-derived gas volume displacement in syringe culture) of C. albicans in a semi-anaerobic environment. (Fig. 1A).

\section{Effect of isoflurane on candidal growth and multiplication} Isoflurane concentrations of $0.1 \%(\mathrm{v} / \mathrm{v})$ and above were inhibitory to yeast multiplication with concentrations of $0.2 \%$ or higher having an effect similar to amphotericin B in a semi-anaerobic environment (Fig. 2). Furthermore, even at the lowest concentration $(0.05 \%)$ liquid isoflurane completely inhibited the formation of hyphae in semi-anaerobic conditions (Fig. 3).

Although lower concentrations $(0.1-0.3 \%)$ of isoflurane revealed inhibitory effects on growth, viability assays of the cultures treated with these concentrations indicated that the yeast were capable of forming hyphae equivalent to control cultures (Figure 4). However, at isoflurane concentrations of $0.3 \%$ and above we observed a significant inhibitory effect on the formation of hyphae. When compared with positive control amphotericin $\mathrm{B}$, a well-characterized and clinically used antifungal agent, a concentration of $0.4 \%$ isoflurane is equivalent to $4 \mu \mathrm{g} / \mathrm{ml}$ of amphotericin $\mathrm{B}$ which is a fungicidal concentration [11]. 
(A)

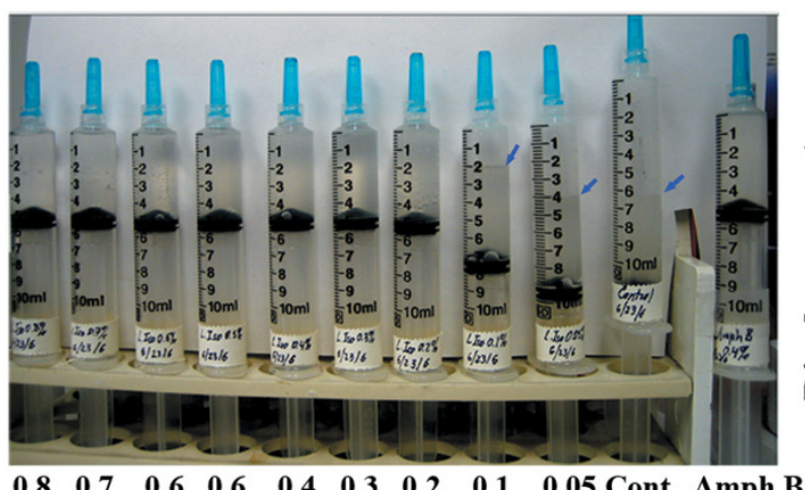

(B)

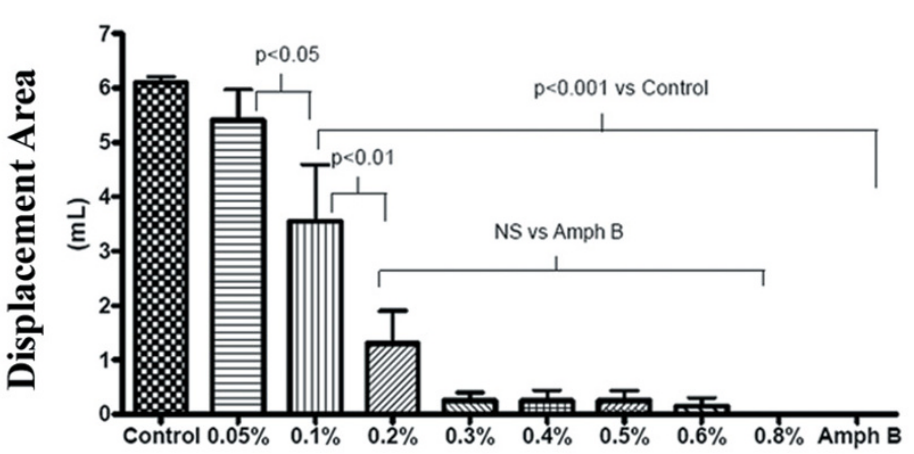

\section{Isoflurane Concentration}

Figure I

Effects of various concentrations of isoflurane on metabolic activity of candidal yeast culture. Actively growing yeast cultures were treated with various concentrations of isoflurane $(0.05 \% 0.8 \% \mathrm{v} / \mathrm{v}$ of media) and amphotericin $\mathrm{B}(4 \mu \mathrm{g} / \mathrm{ml})$. The top of each syringe was tightly sealed to create semi-anaerobic environment. The metabolic activity of each syringe was determined by volume of displacement (an indicator of metabolic activity of Candidal yeast) in the syringe. Panel A shows volume of displacement in syringes (Smaller arrows indicate the levels) whereas Panel B is graphic representation of the displaced volume. Columns in the graph represent the means (+/- Standard error of the mean (SEM)) of four independent experiments.

To further discern whether liquid isoflurane has similar effects on C. albicans growth and morphogenesis under an aerobic environment, Candida yeast cultures treated with various concentrations of liquid isoflurane were allowed to grow at $37^{\circ} \mathrm{C}$ with the syringe caps loosened (completely aerobic environment). The result was that growth of Candida yeast was significantly inhibited at $0.2-0.3 \%$ (Figure 5); however, lower concentrations of isoflurane did not show an inhibitory effect on the formation of hyphae as observed under semi-anaerobic conditions. However, higher concentrations of $0.4 \%$ and above were capable of inhibiting yeast multiplication as well as formation of hyphae as depicted in Figure 6 as well.

However, the non-isoflurane-treated control culture under semi-anaerobic conditions showed the ability to form small hyphae after 16 hrs as shown in Figure 3 (Control). The growth inhibitory effect of $0.4 \%$ isoflurane was equivalent to $4.0 \mu \mathrm{g} / \mathrm{ml}$ of amphotericin B. Both isoflurane and amphotericin $\mathrm{B}$ inhibited formation of hyphae in Candida, which is the form that is commonly associated with pathogenicity.

An interesting observation pertains to the cellular volume of yeast cells. Shrinkage in cellular volume of the yeast was

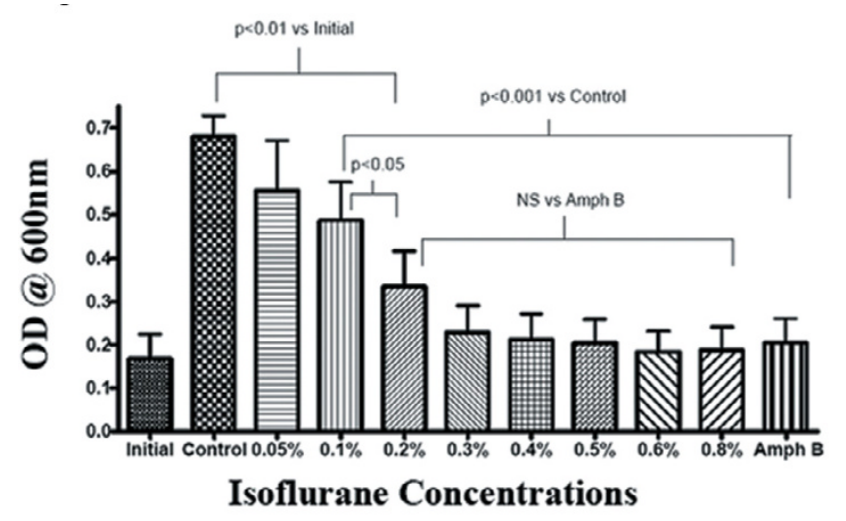

Figure 2

Effects of isoflurane on growth of Candida albicans in semi-anaerobic environment. The effect of various concentrations of isoflurane and anti-fungal Amphotericin B 4 $\mu \mathrm{g} / \mathrm{ml}$ (Amph B) on the growth of syringe culture was determined by optical density (OD) reading at $600 \mathrm{~nm}$. Initial is the OD of starter culture. Columns represent the means (+/-) Standard error of the mean (SEM)) of four independent experiments. NS = Not significant 


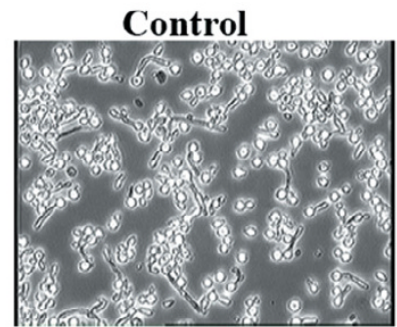

$0.1 \%$ Isoflurane

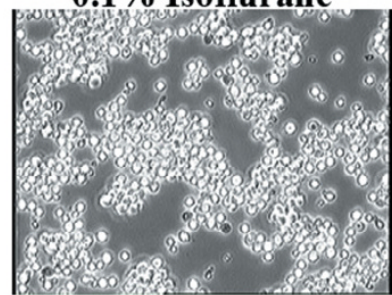

$0.3 \%$ Isoflurane

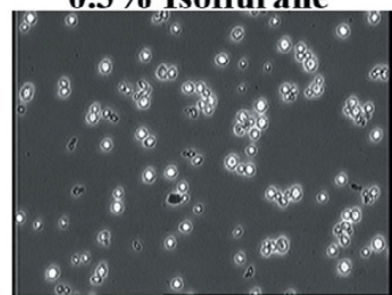

$0.5 \%$ Isoflurane

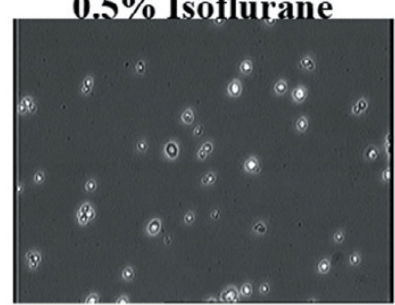

$0.8 \%$ Isoflurane
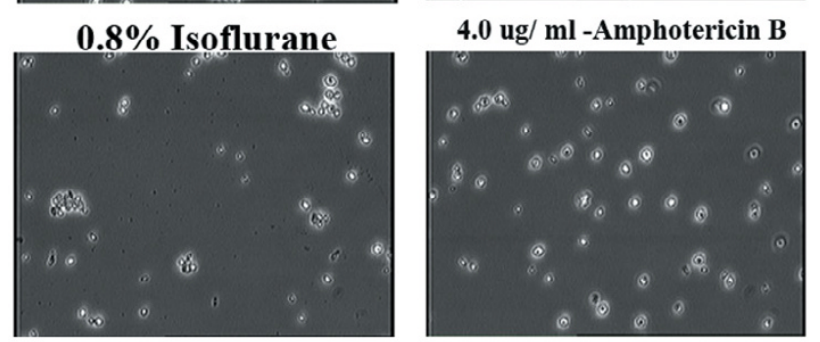

Figure 3

Effects of various concentrations of isoflurane and anti-fungal amphotericin B. Morphological changes in Candida albicans treated with various concentrations of isoflurane and amphotericin $B$ in semi-anaerobic conditions. The magnification of each picture is $60 \times$. The concentrations of isoflurane and amphotericin B are indicated in each panel. Data presented in this figure is representative of four independent experiments.
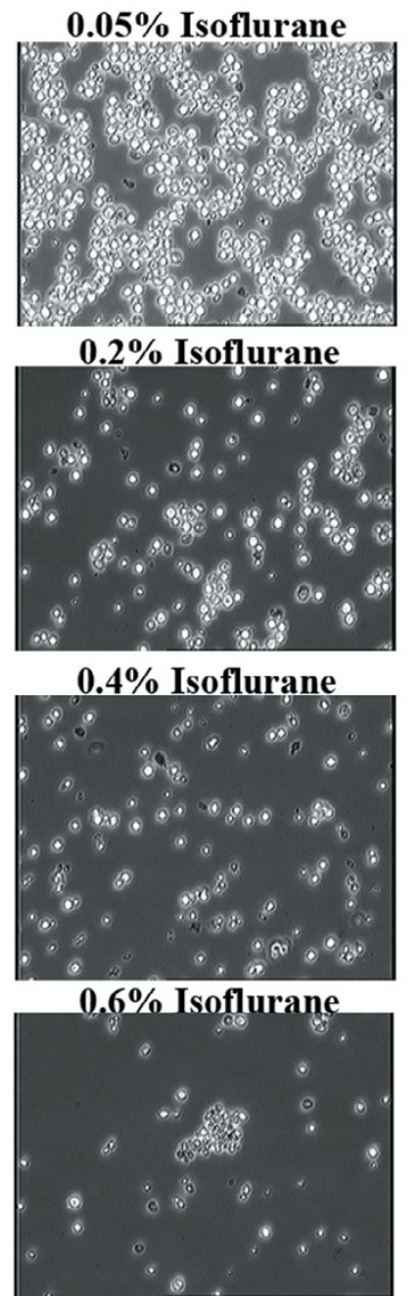

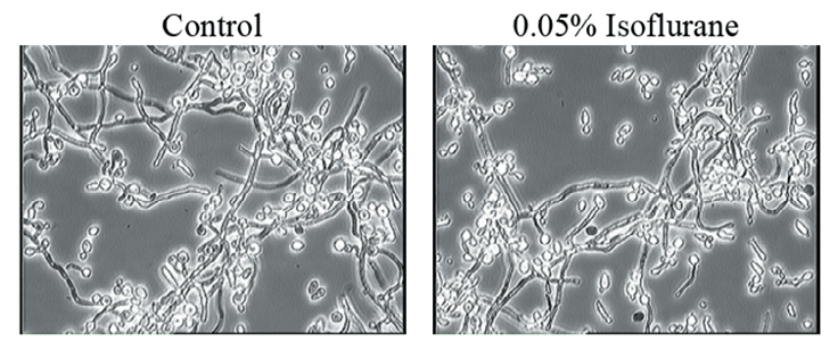

$0.1 \%$ Isoflurane

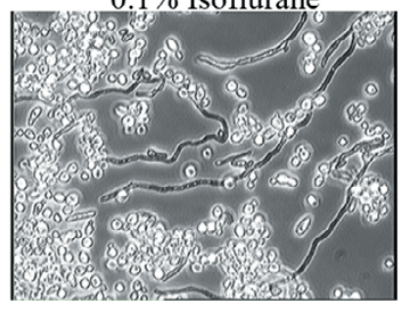

$0.3 \%$ Isoflurane
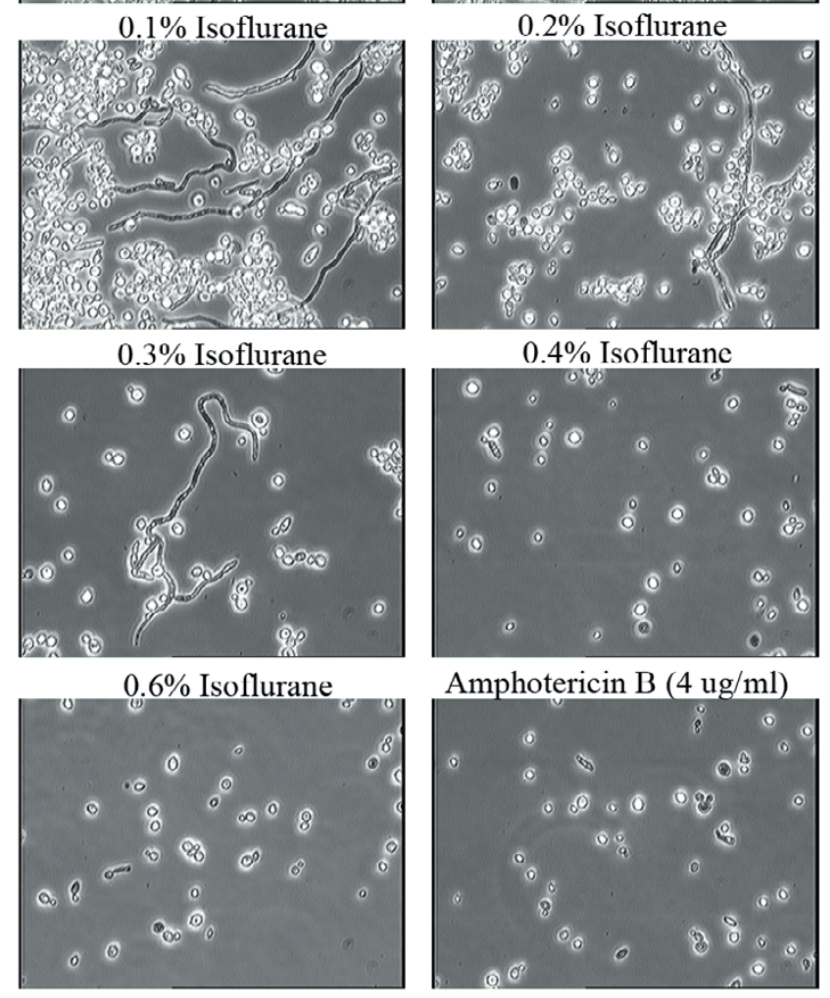

$0.4 \%$ Isoflurane

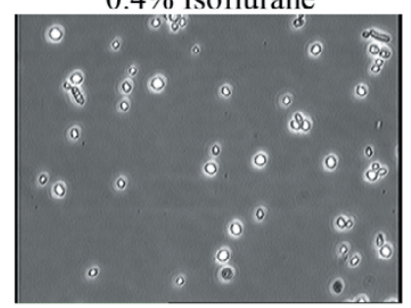

Amphotericin B (4 ug/ml)

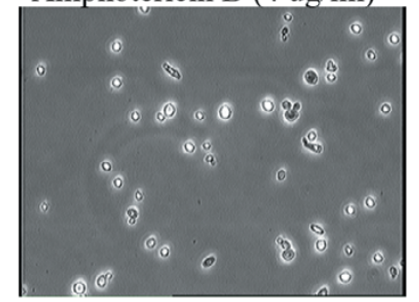

\section{Figure 5}

Morphological changes in Candida albicans treated with various concentrations of isoflurane and amphotericin B in aerobic conditions. The effect of various concentrations of isoflurane and anti-fungal Amphotericin B was determined microscopically. Each culture was thoroughly mixed and $100 \mu \mathrm{l}$ from each syringe was spread on microscopic slides to capture images at $(60 \times)$. Data presented in this figure is representative of three independent experiments.

obvious from cultures treated with isoflurane at a concentration of $0.2 \%$ (Fig. 3). The number of shrunken cells increased with the higher concentrations of isoflurane employed in these experiments. Amphotericin B as described previously also showed cellular shrinkage in our studies $[12,13]$.

\section{Discussion}

Candida albicans is a dimorphic opportunistic pathogen causing both superficial and systemic infections. The most

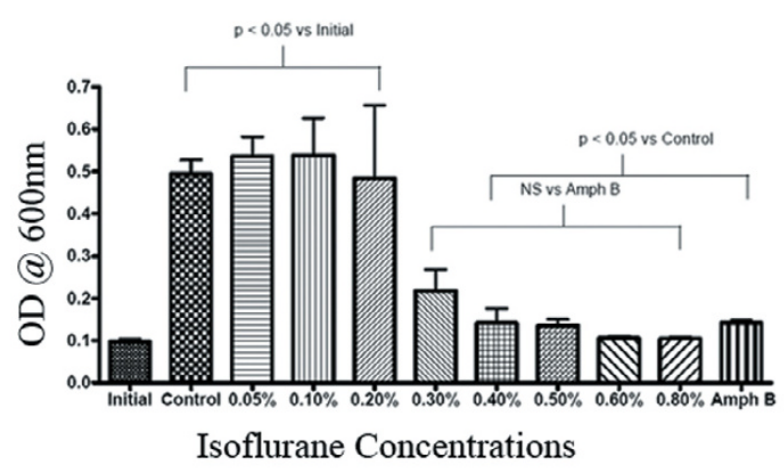

Figure 6

Effects of isoflurane on growth of Candida albicans in aerobic environment. The effect of various concentrations of isoflurane and anti-fungal Amphotericin B on the growth of syringe culture was determined by OD reading at $600 \mathrm{~nm}$. The data presented in this figure is an average of three independent experiments. Columns in the graph represent the means (+/-) Standard error of the mean (SEM).

prominent factors involved in the pathogenesis of this fungus are: capability to exist as both yeast and mycelium with filamentous hyphae (dimorphism) depending on host environment [14]. The hyphal form of this fungus is more invasive and is also involved in the secretion of various proteases and lipases which facilitate tissue invasion of the fungus [2]. Moreover, phenotypic switching between hyphae and yeast form has been proposed as a regulatory element in the pathogenesis of this fungus [15]. In the past, efforts have been directed to identify molecular mechanisms of phenotypic switching between yeast and hyphal morphology of this fungus [16-18].

Like other fungi, an interesting aspect of Candida is its facultative nature [19] and as such can fulfill its energy requirement either by aerobic respiration or anaerobic fermentation. In our studies we determined antifungal activity of liquid isoflurane in both aerobic and semianaerobic environment. Semi-anaerobic environment was created due to the volatile nature of isoflurane, however, in our studies we also used an air-tight chamber and exposed Candida cells with previously described, clinically relevant concentrations of gaseous of isoflurane [13 minimum alveolar concentration (MAC), a term used by anesthetists for defining the strength of anesthetic vapors] that inhibited formation of true hyphae from the yeast (Data not illustrated in this manuscript). Moreover, previous reports suggest that the sensitivity of C. albicans to antifungal drugs particularly Terbinafine, neticonazole and amphotericin B do not vary between aerobic and anaerobic environments [20]. 
Interestingly, liquid isoflurane treatments under aerobic conditions inhibited multiplication of yeast in the same manner as semi-anaerobic environments. However, in aerobic conditions, formation of hyphae were observed in fungal yeast treated with lower concentrations of isoflurane $(0.05-0.2 \%)$ in contrast to semi-anaerobic. It is not clear if this phenotypic difference is due to nuances in toxicity of isoflurane and or different membrane conditions. Despite these differences, higher concentrations of isoflurane showed similar effects under both semi-aerobic and aerobic environments.

As far as growth conditions are concerned, there are few studies describing fungal growth in anaerobic environments in spite of the facultative nature of this fungus. Efforts have been directed towards understanding the survival of C. albicans in anaerobic environments [21,22]. One study describes anaerobic morphogenesis of this fungus in synthetic medium supplemented with yeast extract or a combination of oleic acid, nicotinic acid and ammonium chloride [23]. Although our findings are quite contrary to this study, i.e. limited formation of hyphae in the semi-anaerobic environment was observed. Among dimorphic fungi like Candida, the growth environment plays a major role in the morphogenetic behavior of the fungus. The growth conditions described in our study are quite different than that reported by Dumitru et al [23].

The molecular mechanisms involved in the inhibitory effects of liquid isoflurane on Candida yeast multiplication and hyphae formation require further exploration. It is possible that cellular shrinkage (Please see Figures 3 and 5 ) that could lead to apoptosis of yeast cells is a mechanism by which amphotericin B and isoflurane exert antifungal activity.

Previously, it has been reported that volatile anesthetics exert their effects on living cells by one of the three mechanisms: i) altering the lipid bilayer of the plasma membrane, ii) modulation of membrane bound protein function or iii) a combination of both which involves anesthetic interaction with the lipid bilayer altering activity of membrane proteins [24]. Based on previous observations in Saccharomyces cerevisiae, it is possible that isoflurane might inhibit nutrient-dependent growth either by affecting membrane permeases or mRNA translation $[25,26]$.

C. albicans acts as a silent enemy among healthy individuals and exists mainly by colonizing skin, and mucosal surfaces of oral, digestive tracts and genitalia. Defects in host defense then permit the yeast to promulgate various ailments. Amidst these ailments Candidal vaginitis effects almost 80 percent of healthy women once in their lifetime as well as nearly every immunocompromised woman
$[27,28]$. Moreover, maternal Candida vaginitis can transfer infection to newborn infants in the form of thrush and diaper dermatitis [29]. Development of prophylactic measures of the aforementioned situation would have important public health implications for women and infants. Besides superficial infections, Candida-associated systemic infections are also on the rise, particularly among bone marrow transplant patients who have shown higher mortality associated with fungal infections [30]. Although systemic usage of liquid volatile anesthetic via the intravenous route is associated with adverse effects [31], emulsified isoflurane has been developed and intravenous injections of this formulation has been proven to be safe in animal models [32].

Another interesting aspect of this study is C. albicans dimorphism which has been extensively studied at both cellular and molecular levels [33-35]. The inhibitory effects of isoflurane on formation of hyphae from various Candidal species will provide the opportunity to study the morphological shift in this opportunistic fungus. Furthermore, currently available robust molecular technologies will assist in identifying genetic elements involved in the dimorphic switch.

Our findings show that liquid isoflurane in culture inhibits yeast multiplication as well as formation of hyphae from Candida. It will be essential to further explore the in vivo significance of our in vitro data in order to translate these findings into clinical application. However, in vitro data from our studies strongly suggest that the antifungal activity of liquid isoflurane equals or exceeds Amphotericin B, a gold standard antimycotic currently in use that unfortunately has extremely frequent and serious side effects in humans. The highly lipophilic nature of isoflurane compared to water-soluble Amph B may offer the advantage of better penetration and bioavailability to poorly vascularized tissues. The safety of topically applied liquid isoflurane has been demonstrated in humans [36]. Furthermore, in a canine model, subarachnoid injection of liquid volatile anesthetic agent did not cause permanent motor or sensory neurological injury [37]. Further investigations related to our findings are warranted.

\section{Materials and methods \\ Fungal strain and materials}

We used C. albicans strain H317, a clinical isolate from the Centers for Disease Control and Prevention (Atlanta, GA), that has been described previously [38]. Isoflurane, USP grade was purchased from Baxter (Baxter Healthcare Corporation, Deerfield, IL).

\section{Growth media}

C. albicans H317 cultures were grown in Lee's medium [39] and maintained in $1 \%$ yeast extract, $2 \%$ peptone and 
$2 \%$ dextrose (YPD) medium. Fresh Lee's medium was prepared bi-weekly and stored at $4^{\circ} \mathrm{C}$. An isolated C. albicans colony growing on YPD plate was transferred into $20 \mathrm{ml}$ of pre-warmed Lee's medium and allowed to grow at $27^{\circ} \mathrm{C}$ for initiation of each starter culture.

\section{Effects of liquid isoflurane on metabolic activity of $\mathrm{C}$. albicans}

Actively growing C. albicans yeast cultures at $27^{\circ} \mathrm{C}$ were treated with isoflurane in $10 \mathrm{ml}$ sterile plastic syringes (Becton Dickinson, and Company). Briefly, $5.0 \mathrm{ml}$ of fungal suspensions were treated with isoflurane to final concentrations ranging between $0.05 \%-0.8 \%(\mathrm{v} / \mathrm{v})$. Upon addition of isoflurane the syringe plunger and the top cover were tightly closed and shaken for 5 seconds. As a positive control, one of the culture syringes was treated with amphotericin B $(4.0 \mu \mathrm{g} / \mathrm{ml})$, an antifungal agent widely used in clinical practice. Negative control consisted of Candida yeast culture without any treatment. All syringes were tightly capped to create a semi-anaerobic environment and were transferred to $37^{\circ} \mathrm{C}$ for 16 hours with orbital shaking at $125 \mathrm{rpm}$. For experiments involving aerobic conditions, the syringe caps were loosened to allow air exchange without risk of external contamination.

Effects of various concentrations of isoflurane and amphotericin B on the metabolic activity of fungi were assessed by volume displaced in the syringes under semianaerobic conditions. The volume of displacement from the fungal metabolic activity was recorded with a 3.2 mega pixel digital camera (Figure 1). Upon experiment completion, the optical density (OD) of each culture was also recorded at $600 \mathrm{~nm}$. The effects of various concentrations of isoflurane on the morphology of $C$. albicans were recorded by morphological observations.

\section{Isoflurane and viability of $C$. albicans}

The viability of C. albicans upon treatment with isoflurane for $16 \mathrm{hrs}$ in semi-anaerobic and aerobic environments was determined by adding $0.1 \mathrm{ml}$ of culture from the syringes culture in $2 \mathrm{ml}$ of pre-warmed Lee's medium in $15 \mathrm{ml}$ culture tubes. These tubes were shaken on a rotary shaker for further $22 \mathrm{hrs}$ at $37^{\circ} \mathrm{C}$ in cell-culture tubes. Each culture was thoroughly mixed and $20 \mu \mathrm{l}$ from each syringe was spread on microscopic slides to capture live images (Olympus inverted microscope with monitor and digital camera, Olympus, Japan). Viability as well as morphological changes were assessed and recorded digitally.

\section{Statistical methods}

All quantitative values are presented as means \pm standard error of the mean (SEM). Data comparing fungal metabolism and fungal growth under different conditions were subjected to ANOVA followed by Tukey-Kramer multiple comparisons test. GraphPad statistical software (GraphPad Software, Inc., San Diego, CA) was used to perform the data analysis. Values of $\mathrm{p}<0.05$ were considered statistically significant.

\section{Acknowledgements}

This work was mainly supported by the Department of Anesthesiology Translational Research Funds to VA, Diabetes Trust Foundation Award to MM, and with a partial support from NIH award MH074359 to ZP.

\section{References}

I. Buchheidt D, Skladny H, Baust C, Hehlmann R: Systemic infections with Candida sp. and Aspergillus sp. in immunocompromised patients with hematological malignancies: current serological and molecular diagnostic methods. Chemotherapy 2000, 46(3):219-228.

2. Felk A, Kretschmar M, Albrecht A, Schaller M, Beinhauer S, Nichterlein T, Sanglard D, Korting HC, Schafer W, Hube B: Candida albicans hyphal formation and the expression of the Efglregulated proteinases Sap4 to Sap6 are required for the invasion of parenchymal organs. Infect Immun 2002, 70(7):3689-3700.

3. Hausauer DL, Gerami-Nejad M, Kistler-Anderson C, Gale CA: Hyphal guidance and invasive growth in Candida albicans require the Ras-like GTPase Rsr I p and its GTPase-activating protein Bud2p. Eukaryot Cell 2005, 4(7): | 273-| 286.

4. Lossinsky AS, Jong A, Fiala M, Mukhtar M, Buttle KF, Ingram M: The histopathology of Candida albicans invasion in neonatal rat tissues and in the human blood-brain barrier in culture revealed by light, scanning, transmission and immunoelectron microscopy. Histol Histopathol 2006, 2 I ( I 0): I 029-I 04 I.

5. Iranzo M, Canizares JV, Sainz-Pardo I, Aguado C, Ponton J, Mormeneo $\mathrm{S}$ : Isolation and characterization of an avirulent Candida albicans yeast monomorphic mutant. Med Mycol 2003, 4 I ( I):43-52.

6. Molliex S, Montravers P, Dureuil B, Desmonts JM: Halogenated anesthetics inhibit Pseudomonas aeruginosa growth in culture conditions reproducing the alveolar environment. Anesth Analg 1998, 86(5): 1075-1078.

7. Asehnoune K, Cruaud P, Paries J, Gorce P, Pourriat JL: Effects of isoflurane on bacterial growth. Eur J Anaesthesiol 2000, I 7(5):289-294.

8. Hoerauf K, Koller C, Vescia F, Metz C, Hobbhahn ]: [Exposure of intensive care personnel to isoflurane in long-term sedation]. Anasthesiol Intensivmed Notfallmed Schmerzther 1995, 30(8):483-487.

9. Sackey PV, Martling CR, Nise G, Radell PJ: Ambient isoflurane pollution and isoflurane consumption during intensive care unit sedation with the Anesthetic Conserving Device. Crit Care Med 2005, 33(3):585-590.

10. Odds FC: Candida and candidosis. 2nd Ed Bailliere Tindall, London, England 1988.

II. Phillips AJ, Sudbery I, Ramsdale M: Apoptosis induced by environmental stresses and amphotericin $B$ in Candida albicans. Proc Natl Acad Sci U S A 2003, I00(24): I 4327-I4332.

12. Ramani R, Chaturvedi V: Flow cytometry antifungal susceptibility testing of pathogenic yeasts other than Candida albicans and comparison with the NCCLS broth microdilution test. Antimicrob Agents Chemother 2000, 44( I 0):2752-2758.

13. Ramani R, Ramani A, Wong SJ: Rapid flow cytometric susceptibility testing of Candida albicans. J Clin Microbiol 1997, 35(9):2320-2324.

14. Torosantucci A, Romagnoli G, Chiani P, Stringaro A, Crateri P, Mariotti S, Teloni R, Arancia G, Cassone A, Nisini R: Candida albicans yeast and germ tube forms interfere differently with human monocyte differentiation into dendritic cells: a novel dimorphism-dependent mechanism to escape the host's immune response. Infect Immun 2004, 72(2):833-843.

15. Liu H: Co-regulation of pathogenesis with dimorphism and phenotypic switching in Candida albicans, a commensal and a pathogen. Int J Med Microbiol 2002, 292(5-6):299-3 I I.

16. Cao F, Lane S, Raniga PP, Lu Y, Zhou Z, Ramon K, Chen J, Liu H: The Flo8 transcription factor is essential for hyphal development 
and virulence in Candida albicans. Mol Biol Cell 2006, I 7(I):295-307.

17. Bahn YS, Staab J, Sundstrom P: Increased high-affinity phosphodiesterase PDE2 gene expression in germ tubes counteracts CAPI-dependent synthesis of cyclic AMP, limits hypha production and promotes virulence of Candida albicans. Mol Microbiol 2003, 50(2):39l-409.

18. Bahn YS, Sundstrom P: CAPI, an adenylate cyclase-associated protein gene, regulates bud-hypha transitions, filamentous growth, and cyclic AMP levels and is required for virulence of Candida albicans. J Bacteriol 200I, I 83( I 0):32II-3223.

19. Schaller M, Borelli C, Korting HC, Hube B: Hydrolytic enzymes as virulence factors of Candida albicans. Mycoses 2005, 48(6):365-377.

20. Matsuki M, Kanatsu H, Watanabe T, Ogasawara A, Mikami T, Matsumoto $\mathrm{T}$ : Effects of antifungal drugs on proliferation signals in Candida albicans. Biol Pharm Bull 2006, 29(5):919-922.

21. Eklund T, Jarmund T: Microculture model studies on the effect of various gas atmospheres on microbial growth at different temperatures. J Appl Bacteriol I983, 55(I): I 19-125.

22. Webster CE, Odds FC: Growth of pathogenic Candida isolates anaerobically and under elevated concentrations of $\mathrm{CO} 2$ in air. J Med Vet Mycol I 987, 25(I):47-53.

23. Dumitru R, Hornby JM, Nickerson KW: Defined anaerobic growth medium for studying Candida albicans basic biology and resistance to eight antifungal drugs. Antimicrob Agents Chemother 2004, 48(7):2350-2354.

24. Keil RL, Wolfe D, Reiner T, Peterson CJ, Riley JL: Molecular genetic analysis of volatile-anesthetic action. Mol Cell Biol I 996, 16(7):3446-3453.

25. Palmer LK, Wolfe D, Keeley JL, Keil RL: Volatile anesthetics affect nutrient availability in yeast. Genetics 2002, 161(2):563-574.

26. Palmer LK, Shoemaker JL, Baptiste BA, Wolfe D, Keil RL: Inhibition of translation initiation by volatile anesthetics involves nutrient-sensitive GCN-independent and -dependent processes in yeast. Mol Biol Cell 2005, 16(8):3727-3739.

27. Beltrame A, Matteelli A, Carvalho AC, Saleri N, Casalini C, Capone S, Patroni A, Manfrin M, Carosi G: Vaginal colonization with Candida spp. in human immunodeficiency virus-infected women: a cohort study. Int J STD AIDS 2006, I7(4):260-266.

28. Trama JP, Adelson ME, Raphaelli I, Stemmer SM, Mordechai E: Detection of Candida species in vaginal samples in a clinical laboratory setting. Infect Dis Obstet Gynecol 2005, I3(2):63-67.

29. Chen CJ, Weng YH, Su LH, Huang YC: Molecular evidence of congenital candidiasis associated with maternal candidal vaginitis. Pediatr Infect Dis J 2006, 25(7):655-656.

30. Brown JM: Fungal infections in bone marrow transplant patients. Curr Opin Infect Dis 2004, 17(4):347-352.

31. Kawamoto M, Suzuki N, Takasaki M: Acute pulmonary edema after intravenous liquid halothane in dogs. Anesth Analg 1992, 74(5):747-752.

32. Zhou JX, Luo NF, Liang XM, Liu J: The efficacy and safety of intravenous emulsified isoflurane in rats. Anesth Analg 2006, 102(I):129-134.

33. Mukhtar M, Logan DA, Kaufer NF: The carboxypeptidase $\mathbf{Y}$ encoding gene from Candida albicans and its transcription during yeast-to-hyphae conversion. Gene 1992, I2I(I): $173-177$.

34. Elorza MV, Murgui A, Sentandreu R: Dimorphism in Candida albicans: contribution of mannoproteins to the architecture of yeast and mycelial cell walls. J Gen Microbiol 1985, I 3 |(9):2209-2216.

35. Mitchell AP: Dimorphism and virulence in Candida albicans. Curr Opin Microbiol I 998, I(6):687-692.

36. Fassoulaki A, Skouteri I, Siafaka I, Sarantopoulos C: Local application of volatile anesthetics attenuates the response to a mechanical stimulus in humans. Can J Anaesth 2005, 52(9):95I-957.

37. Garcia-Fernandez J, Parodi E, Garcia P, Matute E, I AGS, Cediel R, Gilsanz F: Clinical actions of subarachnoid sevoflurane administration in vivo: a study in dogs. BrJ Anaesth 2005, 95(4):530-534.

38. Riggsby WS, Torres-Bauza LJ, Wills JW, Townes TM: DNA content, kinetic complexity, and the ploidy question in Candida albicans. Mol Cell Biol 1982, 2(7):853-862.
39. Lee KL, Buckley HR, Campbell CC: An amino acid liquid synthetic medium for the development of mycelial and yeast forms of Candida Albicans. Sabouraudia 1975, I3(2): 148-153.
Publish with Bio Med Central and every scientist can read your work free of charge

"BioMed Central will be the most significant development for disseminating the results of biomedical research in our lifetime. "

Sir Paul Nurse, Cancer Research UK

Your research papers will be:

- available free of charge to the entire biomedical community

- peer reviewed and published immediately upon acceptance

- cited in PubMed and archived on PubMed Central

- yours - you keep the copyright

Submit your manuscript here:

http://www.biomedcentral.com/info/publishing_adv.asp
BiolMedcentral 